\title{
Skull base chondrosarcoma radiosurgery: report of the North American Gamma Knife Consortium
}

\author{
Hideyuki Kano, MD, PhD, ${ }^{1}$ Jason Sheehan, MD, PhD, ${ }^{2}$ Penny K. Sneed, MD, ${ }^{3}$ \\ Heyoung L. McBride, MD, ${ }^{4}$ Byron Young, MD, ${ }^{5}$ Christopher Duma, MD, ${ }^{6}$ David Mathieu, MD, ${ }^{7}$ \\ Zachary Seymour, MD, ${ }^{3}$ Michael W. McDermott, MD, ${ }^{3}$ Douglas Kondziolka, MD, ${ }^{8}$ Aditya lyer, MD, ${ }^{9}$ \\ and L. Dade Lunsford, MD'
}

\begin{abstract}
'Department of Neurological Surgery, University of Pittsburgh, Pittsburgh, Pennsylvania; ${ }^{2}$ Department of Neurological Surgery, University of Virginia, Charlottesville, Virginia; ${ }^{2}$ Departments of Radiation Oncology and Neurological Surgery, University of California, San Francisco, San Francisco, California; ${ }^{4}$ Departments of Radiation Oncology and Neurological Surgery, Barrow Neurological Institute, Phoenix, Arizona; ${ }^{5}$ Department of Neurological Surgery, University of Kentucky, Lexington, Kentucky; ${ }^{6}$ Department of Neurological Surgery, Hoag Memorial Hospital, Newport Beach, California; ${ }^{7}$ Department of Neurological Surgery, Université de Sherbrooke, Centre de Recherche Clinique Étienne-LeBel, Sherbrooke, Quebec, Canada; ${ }^{8}$ Department of Neurological Surgery, New York University Langone Medical Center, New York, New York; and ${ }^{~}$ Department of Neurological Surgery, Stanford University, Stanford, California
\end{abstract}

OBJECT Stereotactic radiosurgery (SRS) is a potentially important option for patients with skull base chondrosarcomas. The object of this study was to analyze the outcomes of SRS for chondrosarcoma patients who underwent this treatment as a part of multimodality management.

METHODS Seven participating centers of the North American Gamma Knife Consortium (NAGKC) identified 46 patients who underwent SRS for skull base chondrosarcomas. Thirty-six patients had previously undergone tumor resections and 5 had been treated with fractionated radiation therapy (RT). The median tumor volume was $8.0 \mathrm{~cm}^{3}$ (range $0.9-28.2 \mathrm{~cm}^{3}$ ), and the median margin dose was $15 \mathrm{~Gy}$ (range 10.5-20 Gy). Kaplan-Meier analysis was used to calculate progression-free and overall survival rates.

RESULTS At a median follow-up of 75 months after SRS, 8 patients were dead. The actuarial overall survival after SRS was $89 \%$ at 3 years, $86 \%$ at 5 years, and $76 \%$ at 10 years. Local tumor progression occurred in 10 patients. The rate of progression-free survival (PFS) after SRS was $88 \%$ at 3 years, $85 \%$ at 5 years, and $70 \%$ at 10 years. Prior RT was significantly associated with shorter PFS. Eight patients required salvage resection, and 3 patients (7\%) developed adverse radiation effects. Cranial nerve deficits improved in $22(56 \%)$ of the 39 patients who deficits before SRS. Clinical improvement after SRS was noted in patients with abducens nerve paralysis $(61 \%)$, oculomotor nerve paralysis $(50 \%)$, lower cranial nerve dysfunction (50\%), optic neuropathy $(43 \%)$, facial neuropathy $(38 \%)$, trochlear nerve paralysis $(33 \%)$, trigeminal neuropathy $(12 \%)$, and hearing loss (10\%).

CONCLUSIONS Stereotactic radiosurgery for skull base chondrosarcomas is an important adjuvant option for the treatment of these rare tumors, as part of a team approach that includes initial surgical removal of symptomatic larger tumors. http://thejns.org/doi/abs/10.3171/2014.12.JNS132580

KEY WORDS chondrosarcoma; Gamma Knife; stereotactic radiosurgery; skull base tumor; oncology

$\mathrm{C}$ HONDROSARCOMAS of the skull base are relatively slow growing and locally invasive tumors that usually do not metastasize distantly until very late in their natural history. ${ }^{10}$ The typical sites of origin are synchondroses of the clivus, the sphenoid-ethmoid re- gion, the parasellar area, and the temporal-occipital junction. ${ }^{23,26}$ The imaging features and clinical presentations of patients with chordomas or chondrosarcomas are similar. Whereas chordomas tend to cause brainstem compression because they arise from the clivus, chondrosarcomas tend

ABBREVIATIONS ARE = adverse radiation effects; EORTC = European Organization for Research and Treatment of Cancer; NAGKC = North American Gamma Knife Consortium; PFS = progression-free survival; PTV = (SRS) planning tumor volume; RT = fractionated radiation therapy; RTOG = Radiation Therapy Oncology Group; SRS = stereotactic radiosurgery; UPMC = University of Pittsburgh Medical Center.

SUBMITTED November 20, 2013. ACCEPTED December 10, 2014.

INCLUDE WHEN CITING Published online June 26, 2015; DOI: 10.3171/2014.12.JNS132580.

DISCLOSURE Drs. Lunsford and Kondziolka are consultants for AB Elekta, and Dr. Lunsford is a stockholder. 
to affect lower cranial nerves as they frequently originate from the occipitotemporal bone synchondrosis. ${ }^{32}$ Due to their location and local invasiveness, it is rare for chondrosarcomas to be completely resectable without additional morbidity. Further management options must be considered for residual tumors. ${ }^{29,30}$ Due to their low vascularity, the presence of extracellular matrix, and their lower mitotic rate, chondrosarcomas had been considered less responsive to conventional photon-based fractionated radiation therapy (RT), the role of which requires additional study. ${ }^{11}$ Advances in photon-based RT have enabled improved dose conformality to a defined target volume and increased sparing of adjacent normal tissue. Combs et al. ${ }^{6}$ recently reported that excellent results (5year progression-free survival [PFS] rates of $85 \%-95 \%$ without cranial nerve deficit or brainstem damage) have been achieved in current series in which patients were treated with photon-based RT with doses ranging from 66 to $76 \mathrm{~Gy}$. They also reported that the 5-year control rate after proton therapy for chondrosarcoma is in the range of $85 \%-95 \%$, albeit with some toxicity. Stereotactic radiosurgery (SRS) has been used as a minimally invasive primary, adjuvant, or salvage option for a relatively few patients with chondrosarcomas, primarily those with smaller tumor volumes. 5,13,17,20,22 The North American Gamma Knife Consortium (NAGKC) was established to evaluate outcomes of SRS for conditions that are relatively rare (e.g., chordoma ${ }^{18}$ cluster headache, ${ }^{19}$ glomus tumors ${ }^{31}$ hemangioblastomas) and to facilitate prospective clinical trials. This report is based on retrospective data provided by 7 medical centers that participate in the NAGKC. In the present study, we analyze the outcomes of SRS for patients who underwent this treatment as a part of multimodality management for skull base chondrosarcomas.

\section{Methods}

\section{Patient Population}

Seven medical centers with individual internal review board approvals for retrospective NAGKC clinical outcome studies participated: the University of Pittsburgh Medical Center (UPMC, 23 patients); University of Virginia (7 patients); University of California, San Francisco (5 patients); Barrow Neurological Institute (7 patients); University of Kentucky (3 patients); Hoag Memorial Hospital (1 patient); and the University of Sherbrooke (1 patient). The records of consecutive skull base chondrosarcoma patients who underwent Gamma Knife SRS between 1988 and 2009 were assessed by each center for inclusion. A database with selected variables was created and sent to all participating centers. Participating centers reviewed the medical records of these patients, entered the data in the spreadsheet, and removed all patient identifiers form the data. These de-identified data were sent to the NAGKC data coordinating center (UPMC) by December 2011. All living patients had a minimum follow-up of 6 months. Clinical and imaging outcomes were assessed at a median follow-up period of 75.4 months (range 6.3-138.5 months) after SRS. Six patients (13\%) had less than 12 months of follow-up after SRS, and 24 patients (52\%) had more than 5 years of follow-up after SRS.
This series included 26 female patients (57\%) and 20 male patients (43\%). Their median age was 43 years (range 15-75 years). Thirty-six patients had prior resections, and 5 had prior fractionated external beam radiation therapy (RT). The median total dose of RT was 54.1 Gy (range 50.4-60 Gy). Thirty-nine patients had their tumor confirmed by biopsy or resection before SRS. Two patients had their chondrosarcoma confirmed at the time of resection performed because of progression despite SRS. Five patients had chondrosarcomas identified by imaging alone. The locations of tumor in these 5 patients without histological confirmation by resection or biopsy included anterior skull base in 2, posterior skull base in 1, petroclival region in 1, and cavernous sinus in 1. Chondrosarcomas were distinguished from chordomas in these 5 patients due to increased bone destruction and tumor mineralization using MRI or CT that allowed delineation from chordoma. ${ }^{13,27}$ Ten patients underwent SRS for recurrent tumors, 26 for residual tumors, and 10 for tumors newly diagnosed by biopsy $(\mathrm{n}=3)$, subsequent resection after SRS ( $n=2)$, or neuroimaging criteria $(n=5)$. The number of prior surgical procedures (1-3) and the tumor locations are shown in Table 1.

\section{Radiosurgery Technique}

Patients underwent application of an imaging-compatible stereotactic head frame with local anesthesia supplemented by intravenous conscious sedation. Highresolution MRI and/or CT was then performed. Patients underwent either sagittal scout MRI or a 3D localizer sequence that included axial, coronal, and sagittal images. A contrast-enhanced spoiled gradient recalled acquisition in steady state (SPGR) sequence was then used to image the tumor and surrounding brain. T2-weighted MR images using fast spin echo technique and bone-window axial CT images also were acquired to assess the infiltrative tumor volume. The target volume included enhanced and nonenhanced tumor regions. A Model U, B, C, or 4-C Leksell Gamma Knife (Elekta Inc.) was used for SRS, depending on the technology available at participating centers. Our radiosurgical technique has been described in detail in previous reports. ${ }^{18}$ In all cases, the radiosurgery treatment volume determined at the time of dose planning conformed to the enhancing tumor volume. The median target volume was $8.0 \mathrm{~cm}^{3}$ (range $0.9-28.2 \mathrm{~cm}^{3}$ ), and the median margin dose was 15 Gy (range 10.5-20 Gy) (Table 2).

\section{Evaluation Criteria}

Clinical and MRI evaluations were performed at intervals of 3-6 months. The follow-up MR images were compared with the intraoperative images, and tumor dimensions were measured in the axial, sagittal, and coronal planes. A volume was then calculated using the reduced formula of an oblate spheroid: XYZ/2. ${ }^{18}$ A complete response was defined as the complete disappearance of enhancing or nonenhancing tumor, a partial response was defined as $>25 \%$ shrinkage of the tumor volume, stable disease was defined as $\pm 25 \%$ change in tumor volume and progressive disease was defined as $>25 \%$ increase in volume of the enhancing or nonenhancing tumor. Tumor 
TABLE 1. Demographic characteristics of the patient population

\begin{tabular}{|c|c|}
\hline Characteristic & No. of Pts \\
\hline \multicolumn{2}{|l|}{ Sex } \\
\hline Male & 20 \\
\hline Female & 26 \\
\hline \multicolumn{2}{|l|}{ Prior surgical procedures } \\
\hline Biopsy & 3 \\
\hline 1 craniotomy & 28 \\
\hline 2 craniotomies & 5 \\
\hline 3 craniotomies & 3 \\
\hline \multicolumn{2}{|l|}{ Tumor location } \\
\hline Clivus & 6 \\
\hline Cavernous sinus & 9 \\
\hline Cavernous sinus-sella-clivus & 1 \\
\hline Cavernous sinus-sphenoid & 1 \\
\hline Cavernous sinus-petroclival & 3 \\
\hline Petroclival & 12 \\
\hline Sphenoid wing & 5 \\
\hline Anterior skull base & 4 \\
\hline Posterior skull base & 3 \\
\hline Occiput-cerebellum & 1 \\
\hline Parietooccipital & 1 \\
\hline \multicolumn{2}{|l|}{ No prior RT group } \\
\hline No prior surgery & 7 \\
\hline Biopsy w/o prior RT & 3 \\
\hline Residual w/o prior RT & 23 \\
\hline Recurrence w/o prior RT & 8 \\
\hline \multicolumn{2}{|l|}{ Prior RT group } \\
\hline Residual w/ prior RT & 3 \\
\hline Recurrence w/ prior RT & 2 \\
\hline
\end{tabular}

Pts = patients; $\mathrm{RT}$ = fractionated radiation therapy; $\mathrm{SRS}=$ stereotactic radiosurgery.

growth within the planned treatment volume was considered treated tumor progression. Tumor growth adjacent to the planned treatment volume but within the $20 \%$ isodose volume was defined as adjacent (marginal) progression. Tumor progression beyond the $20 \%$ isodose volume were considered remote tumor progression. All patients were evaluated for cranial nerve symptoms and signs in the neurosurgery and ophthalmology clinics of each institution. Visual acuity, visual field, and ocular motility was evaluated by ophthalmologists. Any type of improvement in cranial nerve symptoms or signs was considered clinical improvement. Adverse radiation effects (ARE) were evaluated according to the toxicity criteria of the Radiation Therapy Oncology Group (RTOG) and the European Organization for Research and Treatment of Cancer (EORTC) ${ }^{7}$

For statistical analysis, PFS and actuarial overall survival times were calculated from the day of the first SRS using the Kaplan-Meier method. The endpoints of patient survival were the day of death or the last follow-up. The endpoints of PFS were the day of tumor progression confirmed or the last imaging follow-up. Univariate analysis was performed on the Kaplan-Meier curves using logrank statistics with $\mathrm{p}<0.05$ set as significant. Standard statistical processing software (SPSS, version 20.0; IBM Corp.) was used.

\section{Results \\ Patient Survival}

At the time of this assessment, 38 patients (83\%) were alive, and 8 patients $(17 \%)$ had died. Six patients died due to tumor progression, and 2 patients died of unknown causes. The actuarial overall survival since diagnosis for the entire series $(n=46)$ was $98 \%$ at 3 years, $89 \%$ at 5 years, and $75 \%$ at 10 years (Fig. 1). The median interval between initial diagnosis and SRS was 2.8 months (range 0.03-313 months). The overall survival rate after SRS for the entire series (n $=46$ ) was $89 \%$ at 3 years, $86 \%$ at 5 years, and $76 \%$ at 10 years (Fig. 1). None of the assessed variables (age, sex, symptom duration before SRS, duration from diagnosis to SRS, number of prior surgical procedures, prior RT, presence of cranial nerve deficits, tumor volume, and margin dose) were associated with overall survival for the entire series. The overall survival rate after SRS in patients without prior RT $(\mathrm{n}=41)$ was $94 \%$ at 3 years, $91 \%$ at 5 years, and $80 \%$ at 10 years. In the group of patients without prior RT, larger tumor volume (continuous data) was significantly associated with worse overall survival $(\mathrm{p}=0.049$, hazard ratio [HR] 1.07, 95\% CI 1.00-1.14). In the group of patients without prior RT, the 5-year overall survival rate was $100 \%$ when the tumor volume was $<5 \mathrm{~cm}^{3}$ compared with $84 \%$ for patients with tumor volumes $\geq 5 \mathrm{~cm}^{3}$ ( $\mathrm{p}=$ 0.049) (Fig. 1). The overall survival rate in patients with residual tumor and no prior RT $(\mathrm{n}=33)$ was $93 \%$ at 3 and 5 years and $81 \%$ at 10 years after SRS. The overall survival rate in patients with recurrent tumor and no prior RT ( $\mathrm{n}=$ 8) was $100 \%$ at 3 years and $75 \%$ at 5 and 10 years after SRS. Recurrent tumors were not associated with patient survival $(p=0.977)$. Other variables (age, sex, symptom duration between before SRS, duration from diagnosis to SRS, number of prior surgical procedures, presence of cranial nerve deficits, and margin dose) were not associated with overall survival in patients without prior RT.

When the 5 patients without histological confirmation by resection or biopsy were excluded, the overall survival rate after SRS was $88 \%$ at 3 years, $85 \%$ at 5 years, and $74 \%$ at 10 years. There was no statistically significant difference between overall survival in patients with histological confirmation $(n=41)$ and overall survival in patients without histological confirmation $(n=5)$.

\section{Tumor Response}

Five patients developed tumor progression within the SRS planning tumor volume (PTV). Four patients developed tumor progression within the PTV and marginal recurrence. One patient developed tumor progression within the PTV and distant tumor progression at 18 months after SRS. Two patients developed distant tumor progression despite no treated tumor progression at 11 and 136 months after SRS. In the PTV, a total of 10 patients (22\%) developed tumor progression, 21 (46\%) had stable disease, and 15 (33\%) had tumor regression. 
TABLE 2. Patient and radiosurgery treatment characteristics*

\begin{tabular}{lccc}
\hline \multicolumn{1}{c}{ Characteristic } & Prior RT Group $(\mathrm{n}=5)$ & No Prior RT Group $(\mathrm{n}=41)$ & Entire Series $(\mathrm{n}=46)$ \\
\hline Age, yrs & $36.0(35.2-62.5)$ & $42.0(15.3-75.0)$ & $41.7(15.3-75.0)$ \\
\hline Symptom duration before SRS, mos & $14.9(2.0-27.8)$ & $5.8(1.0-152.0)$ & $5.8(1.0-152.0)$ \\
\hline Interval btwn diagnosis \& SRS, mos & $11.5(8.3-94.1)$ & $2.6(0.03-313.1)$ & $2.8(0.03-313.1)$ \\
\hline Interval btwn prior RT and SRS, mos & $4.5(2.7-88.6)$ & NA & $4.5(2.7-88.6)$ \\
\hline Total dose of prior RT, Gy & $54.1(50.4-60)$ & NA & $54.1(50.4-60)$ \\
\hline Follow-up after diagnosis, mos & $90.3(38.5-112.8)$ & $79.9(7.3-331.3)$ & $81.22(7.3-331.3)$ \\
\hline Follow-up after SRS, mos & $17.7(13.0-81.9)$ & $76.4(6.3-138.5)$ & $75.4(6.3-138.5)$ \\
\hline Tumor volume, cm ${ }^{3}$ & $9.9(3.2-21.6)$ & $8.0(0.9-28.2)$ & $8.0(0.9-28.2)$ \\
\hline Margin dose, Gy & $14.5(10.5-16.0)$ & $15.0(11.0-20.0)$ & $15.0(10.5-20.0)$ \\
\hline Maximum dose, Gy & $30.6(21.0-32.0)$ & $30.0(22.0-40.0)$ & $30.0(21.0-40.0)$ \\
\hline Isocenters, $\mathrm{n}$ & $9(3-15)$ & $9(2-25)$ & $9(2-25)$ \\
\hline * Data are presented as median $(\mathrm{range})$ & & &
\end{tabular}

The PFS rate for the entire series was $88 \%$ at 3 years, $85 \%$ at 5 years, and $70 \%$ at 10 years (Fig. 2). Prior RT was significantly associated with shorter PFS $(\mathrm{p}=0.006)$. Other variables (age, sex, symptom duration between before SRS, duration from diagnosis to SRS, number of prior surgical procedures, presence of cranial nerve deficits, histological confirmation, tumor volume, and margin dose) were not associated with PFS for the entire series.

The PFS rate in patients without prior RT was $92 \%$ at 3 years, $88 \%$ at 5 years, and $81 \%$ at 10 years (Fig. 2). In patients without prior RT, larger tumor volume (continuous: $p=0.035$, HR $1.074,95 \%$ CI $1.005-1.147 ; \geq 5 \mathrm{~cm}^{3}$ : $\mathrm{p}=0.043$ ) was significantly associated with shorter PFS. The PFS rate in patients without prior RT and with tumor volume $<5 \mathrm{~cm}^{3}$ was $100 \%$ at 10 years while the PFS rate in patients without prior RT and with tumor volume $\geq 5$ $\mathrm{cm}^{3}$ was $87 \%$ at 3 years, $81 \%$ at 5 years, and $69 \%$ at 10 years (Fig. 2). The PFS rate in patients with residual tumor and no prior RT $(\mathrm{n}=33)$ was $94 \%$ at 3 years, $90 \%$ at 5 years, and $82 \%$ at 10 years after SRS. The PFS rate in patients with recurrent tumor and no prior RT $(n=8)$ was $86 \%$ at 3,5 , and 10 years after SRS. Recurrent tumors were not associated with shorter PFS $(\mathrm{p}=0.977)$. Other variables (age, sex, symptom duration before SRS, duration from diagnosis to SRS, number of prior surgical procedures, presence of $\mathrm{CN}$ deficits, and margin dose) were not determined to be associated with shorter PFS in patients without prior RT.

In the group of patients with residual tumor and no prior RT $(\mathrm{n}=33)$, the PFS rate of patients with a tumor volume $<8 \mathrm{~cm}^{3}(\mathrm{n}=17)$ was $100 \%$ at 10 years while that of patients with a tumor volume $\geq 8 \mathrm{~cm}^{3}(\mathrm{n}=16)$ was $88 \%$ at 3 years, $77 \%$ at 5 years, and $51 \%$ at 10 years after SRS. In patients with residual tumor and no prior RT, tumor volume $<8 \mathrm{~cm}^{3}$ was significantly associated with improved PFS ( $p=0.028)$ (Fig. 3). In the group of patients with residual tumor and no prior RT, the PFS rate of patients with a margin dose $\geq 15$ Gy $(n=22)$ was $100 \%$ at 10 years after SRS while that of patients with a margin dose $<15$ Gy (n $=11$ ) was $82 \%$ at 3 years, $70 \%$ at 5 years, and $47 \%$ at 10 years after SRS. In patients with residual tumor and no prior RT, margin dose $\geq 15$ Gy was significantly associated with improved PFS ( $\mathrm{p}=0.009)$ (Fig. 3).
When 5 patients without histological confirmation by resection or biopsy were excluded, the PFS rate was $87 \%$ at 3 years, $83 \%$ at 5 years, and $74 \%$ at 10 years. There was no statistically significant difference between PFS in patients with histological confirmation $(n=41)$ and those without histological confirmation $(n=5)$. An illustrative case is shown in Fig. 4.

\section{Functional Outcomes}

Longitudinal functional outcomes were available in 44 patients. Twenty-two (56\%) of 39 patients who had cranial nerve deficits before SRS had improvement. The percentages of patients who had improvement in cranial nerve function included: $61 \%$ of those with abducens nerve deficits, $50 \%$ of those with oculomotor nerve deficits, $50 \%$ of those with lower cranial nerve deficits, $43 \%$ of those with optic nerve deficits, $38 \%$ of those with facial nerve deficits, $33 \%$ of those with trochlear nerve deficits, $15 \%$ (12\%) of those with trigeminal nerve deficits, and $10 \%$ of those with hearing deficits (Table 3).

Seven patients had deterioration in cranial nerve function at a median of 4.4 months after SRS (range 1.5-8.3 months) (Table 3). In 1 patient, worsening oculomotor paralysis was due to tumor progression, either in the PTV or adjacent to it 8 months after SRS. Six patients had worsening clinical symptoms in the absence of tumor progression and were considered to have adverse radiation effects (ARE). Four of these patients had tumors that had invaded the cavernous sinus at the time of SRS. Two patients whose tumors were adjacent to the trigeminal nerve or the hypoglossal nerve developed new-onset trigeminal neuralgia and an increase in hypoglossal sensory loss 2.6 months and 4 months, respectively, after SRS. In these patients, we were unable to determine whether microscopic nerve invasion, direct contact by the tumor, or treatmentrelated side effects led to these new symptoms. Symptomatic ARE occurred in 3 patients (7\%), including 2 patients who experienced seizures related to steroid-responsive peritumoral edema and 1 patient who developed a facial neuropathy. None of the patients who developed ARE had undergone prior RT. 

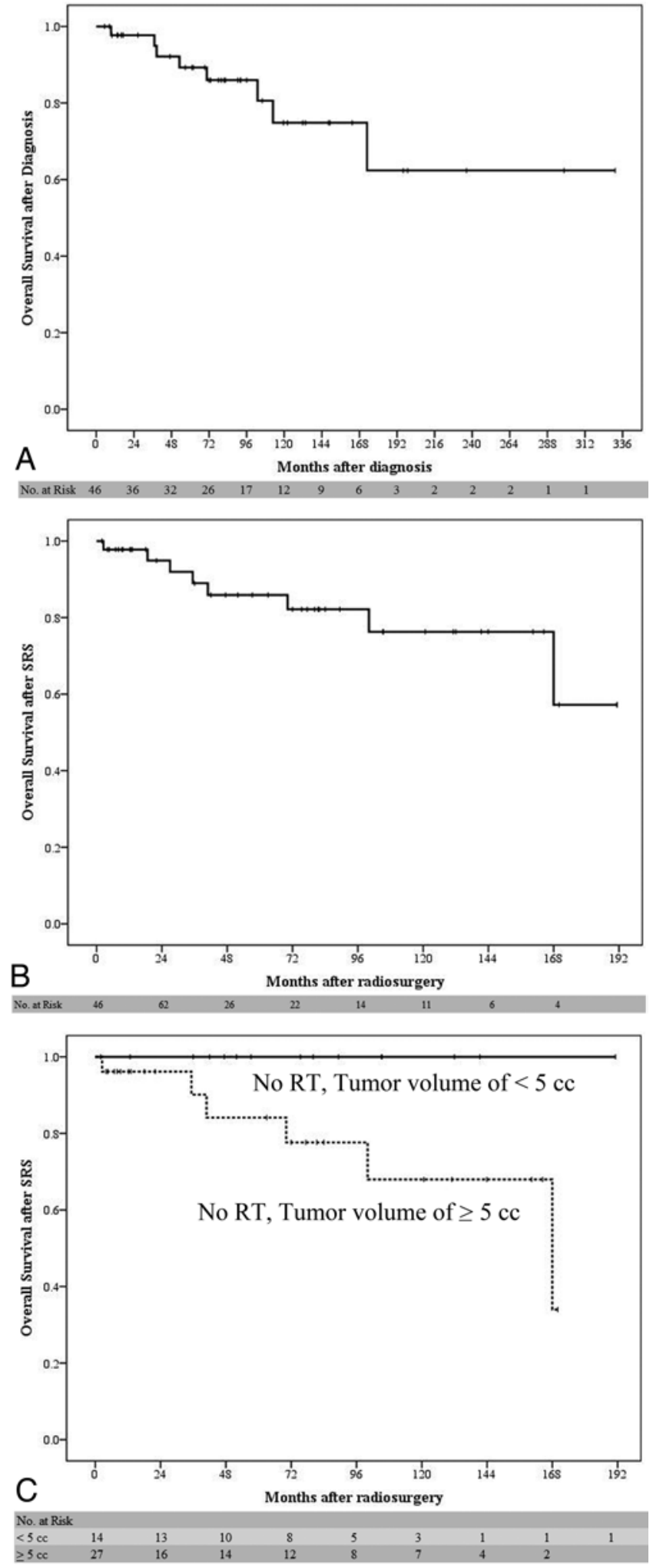

FIG. 1. A: Kaplan-Meier estimate of actuarial overall survival curve in chondrosarcoma patients after diagnosis. B: Kaplan-Meier estimate of actuarial overall survival curve in chondrosarcoma patients after SRS. C: Kaplan-Meier curves comparing overall survival after SRS in patients without prior RT stratified by tumor volume $\left(<5 \mathrm{vs} \geq 5 \mathrm{~cm}^{3}\right)$.
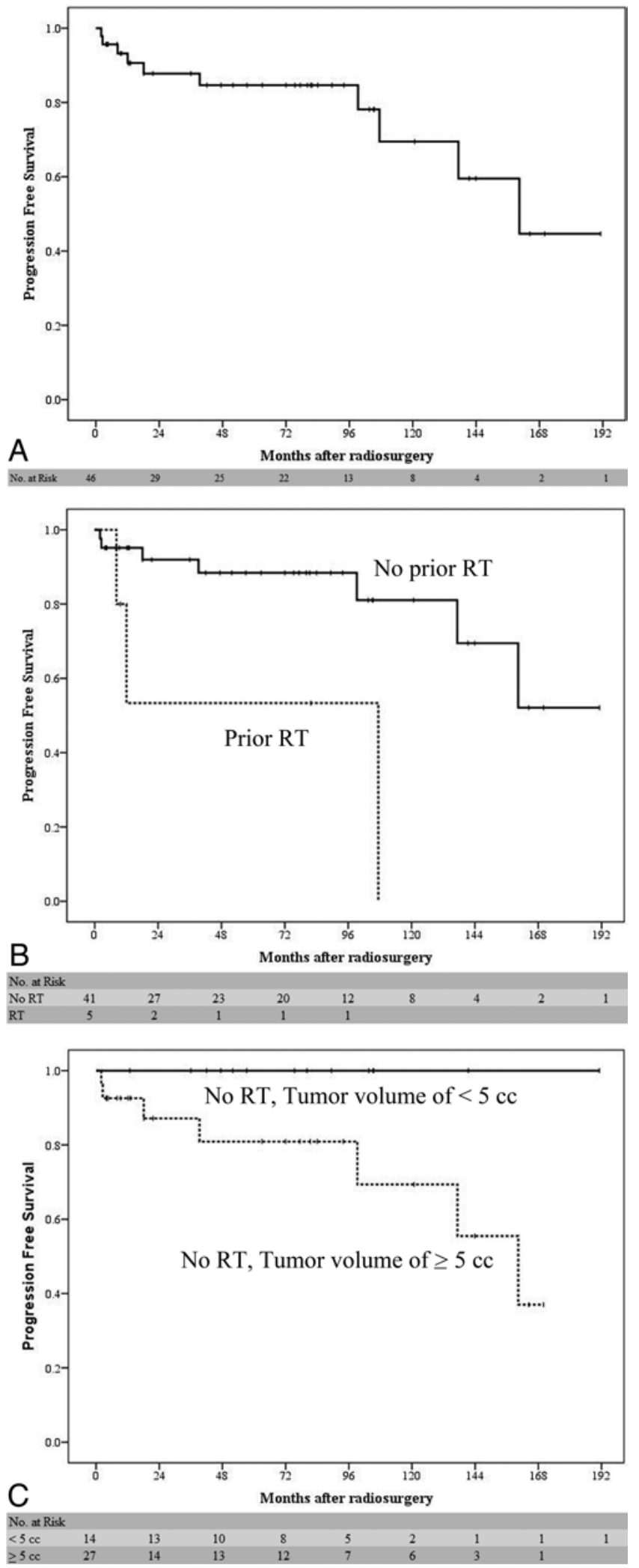

FIG. 2. A: Kaplan-Meier estimate of PFS curve in chondrosarcoma patients after SRS. B: Kaplan-Meier curves comparing PFS after SRS in patients who had previously been treated with RT versus those who had not. C: Kaplan-Meier curves comparing PFS after SRS in patients without prior RT stratified by tumor volume $\left(<5 \mathrm{vs} \geq 5 \mathrm{~cm}^{3}\right)$. 


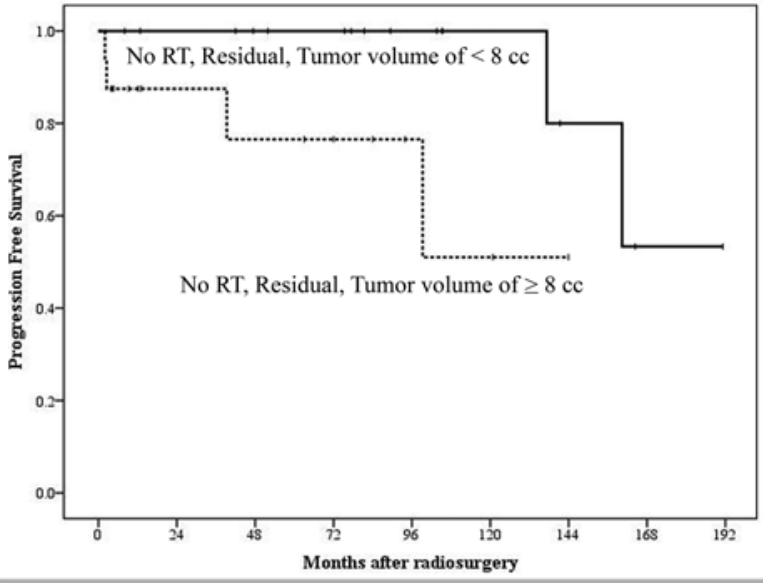

$\begin{array}{llccccccc}\text { No. at Risk } & 17 & 15 & 13 & 12 & 8 & 5 & 3 & 1 \\ <8 \mathrm{cc} & 17 & 8 & 7 & 5 & 3 & 2 & & \end{array}$

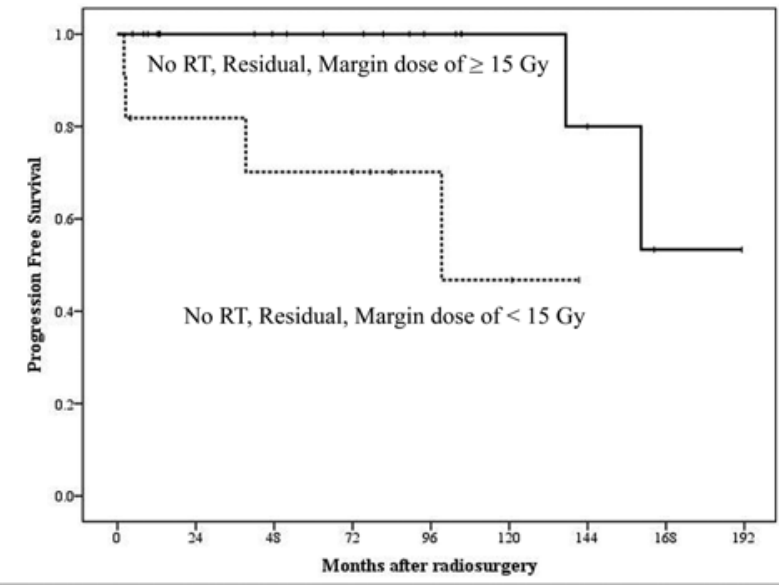

\begin{tabular}{|c|c|c|c|c|c|c|c|c|}
\hline \multicolumn{9}{|l|}{ No. at Risk } \\
\hline$\geq 15 \mathrm{~Gy}$ & 22 & 16 & 14 & 12 & 8 & 5 & 3 & 1 \\
\hline$<15 \mathrm{~Gy}$ & 11 & 7 & 6 & 5 & 3 & 2 & & \\
\hline
\end{tabular}

FIG. 3. Upper: Kaplan-Meier curves comparing PFS after SRS for patients with residual tumor and no prior RT stratified by tumor volume $1<8$ $v S \geq 8 \mathrm{~cm}^{3}$ ). Lower: Kaplan-Meier curves comparing PFS after SRS for patients with residual tumor and no prior RT stratified by tumor volume ( $<15$ vs $\geq 15$ Gy)

\section{Additional Management After Radiosurgery}

Three patients had repeat SRS, 1 patient had fractionated RT 1 month after SRS, and 8 patients underwent subsequent resection due to tumor progression at a median of 53 months after SRS (range 13.5-168 months). Among the 3 patients who had repeat SRS, 1 patient underwent repeat SRS alone for treated tumor progression 101 months after the initial SRS, 1 patient underwent resection followed by postoperative SRS for treated and marginal tumor progression 101 months after the initial SRS, and 1 patient underwent chemotherapy followed by resection and repeat SRS for treated tumor progression 9 months after the initial SRS.

\section{Discussion}

Distinguishing chondrosarcomas from chordomas by imaging alone is not always easy, but it is clinically important, because the prognosis is generally considered rela-
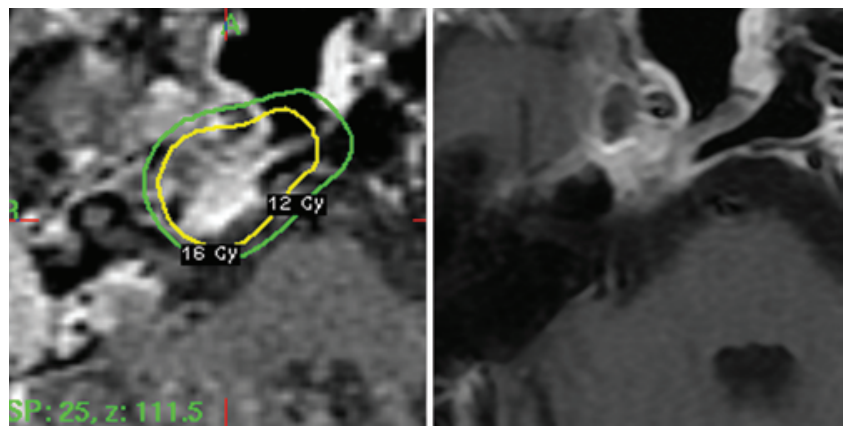

FIG. 4. Left: Axial T1-weighted contrast-enhanced MR image obtained in a 74-year-old woman showing a chondrosarcoma at the time of SRS for treatment of residual tumor following resection. The tumor volume was $10 \mathrm{~cm}^{3}$, and the margin dose was $16 \mathrm{~Gy}$. Right: Axial T1-weighted contrast-enhanced MR image obtained 3 years after SRS showing slight regression of the tumor. Figure is available in color online only.

tively better for chondrosarcomas. ${ }^{12,24}$ The most common presenting symptom in patients with chondrosarcoma is diplopia, secondary to abducens nerve palsy. ${ }^{3}$ In the present study, 23 patients $(50 \%)$ presented with abducens nerve palsy and $16(35 \%)$ presented with trigeminal neuropathy.

\section{Therapeutic Options for Chondrosarcomas}

Chondrosarcomas are rarely completely resectable and additional treatment is generally recommended for subtotal resected tumors. ${ }^{29,30}$ Crockard et al. reported a 93\% 5-year survival rate for 17 patients who underwent surgery as the sole treatment. ${ }^{8}$ In contrast, Bloch et al..$^{4}$ conducted a multireport systematic review of 560 cases of chondrosarcoma and found a recurrence rate of $44 \%$ of patients who underwent resection as the sole treatment, compared with 9\% in patients who had surgery followed by radiation therapy. The 5-year mortality rate was $11.5 \%$, and the median survival was 24 months. The authors found no association between the rate of recurrence and the histological grade of the tumor. ${ }^{4}$ Studies using proton beam RT for chondrosarcomas suggest that the overall survival and local tumor control rates at 5 years are above $90 \% .^{14,21,26}$ Amichetti et al. ${ }^{1}$ reported that the early acute toxicity rates are higher with fractionated proton beam RT. Ares et al. ${ }^{2}$ treated 22 chondrosarcomas using proton beam RT. High-grade late radiation toxicity was found in $6 \%$ of the patients. SchulzErtner et al. ${ }^{28}$ reported that RTOG/EORTC Grade 3 toxicities developed in $4.1 \%$ of 54 chondrosarcoma patients who underwent carbon ion RT. Adverse radiation effects were reported in the range of 5\%-17\% of patients, including patients with RTOG/EORTC Grade 4 toxicities.,14-16,24,25,27 The rate of adverse radiation effects of proton beam RT was higher than that of SRS, because proton beam RT tends to be used for larger tumors compared with SRS. Proton beam RT is expensive and can be provided in only a limited number of institutions. Therefore, it is still not a standard treatment modality for chondrosarcomas. The numbers of patients in other published chondrosarcoma series are small, reflecting the role of surgery, generally followed by photon RT. Such series also describe 90\%$100 \%$ actuarial local control rates at 5 years. ${ }^{9,10}$ 
TABLE 3. Cranial nerve response after radiosurgery

\begin{tabular}{|c|c|c|c|c|c|}
\hline $\begin{array}{l}\text { CNs w/ } \\
\text { Deficits }\end{array}$ & $\begin{array}{c}\text { Before } \\
\text { SRS }\end{array}$ & Improved & $\begin{array}{c}\text { No } \\
\text { Change }\end{array}$ & Worse & Cause of CN Deterioration (tumor location, vol, margin dose) \\
\hline II & 7 & 3 & 3 & 1 & ARE (CS-S-C, $\left.11 \mathrm{~cm}^{3}, 11 \mathrm{~Gy}\right)$ \\
\hline III & 10 & 5 & 3 & 2 & PD (CS, $7.6 \mathrm{~cm}^{3}, 15$ Gy), ARE (CS-S-C, $\left.11 \mathrm{~cm}^{3}, 11 \mathrm{~Gy}\right)$ \\
\hline IV & 3 & 1 & 1 & 1 & ARE (CS-S-C, $\left.11 \mathrm{~cm}^{3}, 11 \mathrm{~Gy}\right)$ \\
\hline V & 16 & 2 & 11 & 3 & ARE (CS-S-C, $\left.11 \mathrm{~cm}^{3}, 11 \mathrm{~Gy}\right)$, ARE (PSB, $\left.1.1 \mathrm{~cm}^{3}, 20 \mathrm{~Gy}\right)$, ARE (PC, $\left.3.4 \mathrm{~cm}^{3}, 16 \mathrm{~Gy}\right)$ \\
\hline $\mathrm{VI}$ & 23 & 14 & 7 & 2 & ARE (CS-S-C, $\left.11 \mathrm{~cm}^{3}, 11 \mathrm{~Gy}\right)$, ARE (PC, $\left.8.3 \mathrm{~cm}^{3}, 16 \mathrm{~Gy}\right)$ \\
\hline VII & 8 & 3 & 5 & 0 & NA \\
\hline VIII & 10 & 1 & 8 & 1 & ARE (ant skull base, $2.3 \mathrm{~cm}^{3}, 15 \mathrm{~Gy}$ ) \\
\hline IX & 4 & 3 & 0 & 1 & ARE (petroclival, $18.4 \mathrm{~cm}^{3}, 18 \mathrm{~Gy}$ ) \\
\hline$X$ & 5 & 3 & 2 & 0 & NA \\
\hline $\mathrm{XI}$ & 5 & 3 & 2 & 0 & NA \\
\hline XII & 6 & 3 & 3 & 0 & NA \\
\hline
\end{tabular}

$\mathrm{ARE}=$ adverse radiation effects; $\mathrm{CN}$ = cranial nerve; $\mathrm{CS}-\mathrm{S}-\mathrm{C}=$ cavernous sinus-sella-clivus; $\mathrm{NA}$ = not applicable; $\mathrm{PC}=$ petroclival; $\mathrm{PD}=$ progression of disease; $\mathrm{PSB}=$ posterior skull base.

\section{Stereotactic Radiosurgery for Chondrosarcomas}

Relatively little data exists to define the use of SRS in the multimodality management of chondrosarcoma. SRS has been shown to result in less toxicity to surrounding structures and have fewer complications than RT in the management of chondrosarcomas. Hasegawa et al. studied 7 chondrosarcoma patients who underwent SRS..$^{13}$ The 5 -year PFS rate was $76 \%$. A tumor volume of less than $20 \mathrm{~cm}^{3}$ was associated with significantly improved PFS. Krishnan et al. reported on 4 patients with chondrosarcomas who underwent SRS and had tumor control at 5 years..$^{22}$ In the present study, the 5-year PFS rate after SRS was $85 \%$. Prior RT was significantly associated with a shorter PFS. In patients without prior RT, a tumor volume of less than $5 \mathrm{~cm}^{3}$ was significantly associated with improved PFS. SRS would be a potent treatment option for small to medium-sized chondrosarcomas before photon or proton-based RT.

Iyer et al. studied 22 patients (these patients are included in the current report) who underwent Gamma Knife SRS for residual or recurrent intracranial chondrosarcomas. ${ }^{17}$ The overall patient survival rates were $95 \%$ at 1 year after SRS, $70 \%$ at 5 years, and $56 \%$ at 10 years. Factors associated with longer survival after SRS included a shorter interval (< 6 months) between diagnosis and SRS, age greater than 40 years, and either a single or no prior resection. The PFS rates were $91 \%$ at 1 year, $72 \%$ at 5 years, and $54 \%$ at 10 years. Factors associated with longer PFS after SRS included patient age greater than 40 years and no prior RT. In the present study, a larger tumor volume in patients without prior RT was significantly associated with worse overall survival. Prior RT was significantly associated with shorter PFS. In patients with residual tumor and no prior RT, tumor volume $<8 \mathrm{~cm}^{3}$ and margin dose $\geq 15$ Gy was significantly associated with improved PFS (Fig. 3). Smaller residual chondrosarcomas were considered to be a good candidate for SRS. The percentage of patients with cranial nerve disorders who reported improved function ranged from $10 \%$ in the case of hearing deficit to $61 \%$ of patients with abducens nerve palsy.

\section{Study Limitations and Future Directions}

The limitations of this study include the inherent differences in patient selection and treatment afforded by a retrospective multicenter study. In addition, the length of follow-up for some of the patients was less than 12 months. making it difficult to determine the effect of treatment versus the natural history of a slow-growing, skull base lesion such as a chondrosarcoma. In a future study, we anticipate an analysis of outcomes for chondrosarcoma patients who undergo repeat SRS. Finally, neuroimaging and radiosurgical techniques have changed over the years. These changes have likely led to earlier detection of residual or recurrent chondrosarcomas following resection and facilitated improvements in radiosurgical delivery.

\section{Conclusions}

Stereotactic radiosurgery provided a reasonable benefit-to-risk profile for patients with residual or newly diagnosed small skull base chondrosarcomas in this multicenter experience. The ability to achieve tumor control of chondrosarcomas is likely enhanced by earlier recognition and the application of multimodal treatment in appropriate patients. Maximal safe resection should be the primary initial management of chondrosarcomas. SRS is a potent treatment option for small to medium-sized chondrosarcomas and is associated with improvement of cranial nerve function in selected cases, especially for patients who present with diplopia related to abducens nerve palsy.

\section{References}

1. Amichetti M, Cianchetti M, Amelio D, Enrici RM, Minniti G: Proton therapy in chordoma of the base of the skull: a systematic review. Neurosurg Rev 32:403-416, 2009

2. Ares C, Hug EB, Lomax AJ, Bolsi A, Timmermann B, Rutz $\mathrm{HP}$, et al: Effectiveness and safety of spot scanning proton radiation therapy for chordomas and chondrosarcomas of the skull base: first long-term report. Int J Radiat Oncol Biol Phys 75:1111-1118, 2009

3. Bloch OG, Jian BJ, Yang I, Han SJ, Aranda D, Ahn BJ, et al: 
Cranial chondrosarcoma and recurrence. Skull Base 20:149156,2010

4. Bloch OG, Jian BJ, Yang I, Han SJ, Aranda D, Ahn BJ, et al: A systematic review of intracranial chondrosarcoma and survival. J Clin Neurosci 16:1547-1551, 2009

5. Cho YH, Kim JH, Khang SK, Lee JK, Kim CJ: Chordomas and chondrosarcomas of the skull base: comparative analysis of clinical results in 30 patients. Neurosurg Rev 31:35-43, 2008

6. Combs SE, Laperriere N, Brada M: Clinical controversies: proton radiation therapy for brain and skull base tumors. Semin Radiat Oncol 23:120-126, 2013

7. Cox JD, Stetz J, Pajak TF: Toxicity criteria of the Radiation Therapy Oncology Group (RTOG) and the European Organization for Research and Treatment of Cancer (EORTC). Int J Radiat Oncol Biol Phys 31:1341-1346, 1995

8. Crockard HA, Cheeseman A, Steel T, Revesz T, Holton JL, Plowman N, et al: A multidisciplinary team approach to skull base chondrosarcomas. J Neurosurg 95:184-189, 2001

9. Debus J, Schulz-Ertner D, Schad L, Essig M, Rhein B, Thillmann $\mathrm{CO}$, et al: Stereotactic fractionated radiotherapy for chordomas and chondrosarcomas of the skull base. Int J Radiat Oncol Biol Phys 47:591-596, 2000

10. Gay E, Sekhar LN, Rubinstein E, Wright DC, Sen C, Janecka IP, et al: Chordomas and chondrosarcomas of the cranial base: results and follow-up of 60 patients. Neurosurgery 36:887-896, 1995

11. Gelderblom H, Hogendoorn PC, Dijkstra SD, van Rijswijk CS, Krol AD, Taminiau AH, et al: The clinical approach towards chondrosarcoma. Oncologist 13:320-329, 2008

12. Goel A: Chordoma and chondrosarcoma: relationship to the internal carotid artery. Acta Neurochir (Wien) 133:30-35, 1995

13. Hasegawa T, Ishii D, Kida Y, Yoshimoto M, Koike J, Iizuka H: Gamma Knife surgery for skull base chordomas and chondrosarcomas. J Neurosurg 107:752-757, 2007

14. Hug EB, Loredo LN, Slater JD, DeVries A, Grove RI, Schaefer RA, et al: Proton radiation therapy for chordomas and chondrosarcomas of the skull base. J Neurosurg 91:432 439, 1999

15. Hug EB, Sweeney RA, Nurre PM, Holloway KC, Slater JD, Munzenrider JE: Proton radiotherapy in management of pediatric base of skull tumors. Int J Radiat Oncol Biol Phys 52:1017-1024, 2002

16. Igaki H, Tokuuye K, Okumura T, Sugahara S, Kagei K, Hata $\mathrm{M}$, et al: Clinical results of proton beam therapy for skull base chordoma. Int J Radiat Oncol Biol Phys 60:11201126,2004

17. Iyer A, Kano H, Kondziolka D, Liu X, Niranjan A, Flickinger JC, et al: Stereotactic radiosurgery for intracranial chondrosarcoma. J Neurooncol 108:535-542, 2012

18. Kano H, Iqbal FO, Sheehan J, Mathieu D, Seymour ZA, Niranjan A, et al: Stereotactic radiosurgery for chordoma: a report from the North American Gamma Knife Consortium. Neurosurgery 68:379-389, 2011

19. Kano H, Kondziolka D, Mathieu D, Stafford SL, Flannery TJ, Niranjan A, et al: Stereotactic radiosurgery for intractable cluster headache: an initial report from the North American Gamma Knife Consortium. J Neurosurg 114:1736-1743, 2011

20. Koga T, Shin M, Saito N: Treatment with high marginal dose is mandatory to achieve long-term control of skull base chordomas and chondrosarcomas by means of stereotactic radiosurgery. J Neurooncol 98:233-238, 2010

21. Korten AG, ter Berg HJ, Spincemaille GH, van der Laan RT, Van de Wel AM: Intracranial chondrosarcoma: review of the literature and report of 15 cases. J Neurol Neurosurg Psychiatry 65:88-92, 1998

22. Krishnan S, Foote RL, Brown PD, Pollock BE, Link MJ,
Garces YI: Radiosurgery for cranial base chordomas and chondrosarcomas. Neurosurgery 56:777-784, 2005

23. Lee SY, Lim YC, Song MH, Seok JY, Lee WS, Choi EC: Chondrosarcoma of the head and neck. Yonsei Med J 46:228-232, 2005

24. Munzenrider JE, Liebsch NJ: Proton therapy for tumors of the skull base. Strahlenther Onkol 175 (Suppl 2):57-63, 1999

25. Pai HH, Thornton A, Katznelson L, Finkelstein DM, Adams JA, Fullerton BC, et al: Hypothalamic/pituitary function following high-dose conformal radiotherapy to the base of skull: demonstration of a dose-effect relationship using dosevolume histogram analysis. Int J Radiat Oncol Biol Phys 49:1079-1092, 2001

26. Rosenberg AE, Nielsen GP, Keel SB, Renard LG, Fitzek MM, Munzenrider JE, et al: Chondrosarcoma of the base of the skull: a clinicopathologic study of 200 cases with emphasis on its distinction from chordoma. Am J Surg Pathol 23:1370-1378, 1999

27. Santoni R, Liebsch N, Finkelstein DM, Hug E, Hanssens $\mathrm{P}$, Goitein M, et al: Temporal lobe (TL) damage following surgery and high-dose photon and proton irradiation in 96 patients affected by chordomas and chondrosarcomas of the base of the skull. Int J Radiat Oncol Biol Phys 41:59-68, 1998

28. Schulz-Ertner D, Nikoghosyan A, Hof H, Didinger B, Combs SE, Jäkel O, et al: Carbon ion radiotherapy of skull base chondrosarcomas. Int J Radiat Oncol Biol Phys 67:171-177, 2007

29. Sekhar LN, Pranatartiharan R, Chanda A, Wright DC: Chordomas and chondrosarcomas of the skull base: results and complications of surgical management. Neurosurg Focus 10(3):E2, 2001

30. Sen CN, Sekhar LN: The subtemporal and preauricular infratemporal approach to intradural structures ventral to the brain stem. J Neurosurg 73:345-354, 1990

31. Sheehan JP, Tanaka S, Link MJ, Pollock BE, Kondziolka D, Mathieu D, et al: Gamma Knife surgery for the management of glomus tumors: a multicenter study. J Neurosurg 117:246-254, 2012

32. Volpe NJ, Liebsch NJ, Munzenrider JE, Lessell S: Neuroophthalmologic findings in chordoma and chondrosarcoma of the skull base. Am J Ophthalmol 115:97-104, 1993

\section{Author Contributions}

Conception and design: Kano. Acquisition of data: Kano, Sheehan, Sneed, McBride, Young, Duma, Mathieu, Seymour, Iyer. Analysis and interpretation of data: Kano. Drafting the article: Kano, Sheehan, Lunsford. Critically revising the article: all authors. Reviewed submitted version of manuscript: all authors. Approved the final version of the manuscript on behalf of all authors: Kano. Statistical analysis: Kano. Study supervision: Kano.

\section{Supplemental Information}

Previous Publication

This study was presented and awarded the Synthes Skull Base Surgery Award at the 63rd Annual Meeting of the Congress of Neurological Surgeons, San Francisco, California, October 19-23, 2013

\section{Correspondence}

Hideyuki Kano, Department of Neurological Surgery, University of Pittsburgh, Ste. B-400, UPMC Presbyterian, 200 Lothrop St., Pittsburgh,PA 15213. email: kanoh@upmc.edu. 